\title{
Rough terrain profiling using digital image correlation
}

\author{
Theunis R. Botha ${ }^{*} \&$ P. Schalk Els \\ Department of Mechanical and Aeronautical Engineering, \\ University of Pretoria, co Lynnwood Road and Roper Street, \\ Pretoria, 0002, South Africa
}

\footnotetext{
* Corresponding author.

E-mail address: trbotha@tuks.co.za
}

Telephone: +27124203289

E-mail address:

trbotha@tuks.co.za (Theunis R. Botha)

schalk.els@up.ac.za (P. Schalk Els)

\begin{abstract}
Road profiling is an important aspect of vehicle dynamics simulations especially over rough terrains. The accurate measurement of rough terrains allows for more accurate multi body simulations. Three dimensional road profiles are usually performed by utilising a line scan sensor which measures several points lateral to the road. The sensors range from simple road following wheels to LiDAR sensors. The obtained line scans are longitudinally stitched together using the orientation and position of the sensor to obtain a full three dimensional road profile. The sensor's position and orientation therefore needs to be accurately determined in order to combine the line scans to create an accurate representation of the terrain. The sensor's position and orientation is normally measured using an expensive inertial measurement unit or Inertial Navigation System (INS) with high sensitivity, low noise and low drift. This paper proposes a road profiling technique which utilises stereography, based on two inexpensive digital cameras, to obtain three-dimensional measurements of the road. The system negates the use of an expensive INS system to determine orientation and position. The data sets also require
\end{abstract}


subsampling which can be computationally expensive. A simple subsampling routine is presented which takes advantage of the structure of the data sets to significantly speed up the process.

\section{Introduction}

The vertical dynamics of a vehicle is important for analysing the suspension, tyre and fatigue life of a vehicle. The use of computer aided simulation is often used to speed up and reduce the costs of the analysis. The road input is the main source of vertical excitation of a vehicle [1] and therefore requires a realistic representation of road profiles. While some road profiles can easily be represented using standard road profile models [2] extremely rough surfaces used in agricultural or off-road terrain cannot. The accurate measurement of real terrain surfaces not only provides a realistic representation of the desired terrain on which the vehicle will operate, but can also serve to be used as validation between the measurement and simulation.

In terramechanics soil deformation is used not only to evaluate the performance of wheels in deformable soil [3] but also used to improve soil models for simulation and prediction [4]. Accurate measurement of the deformed soil can improve the development for off-road terrain wheels. Improved off-road terrain wheels would lead not only to improved tractive performance of the wheels but also decrease the rutting of soils. The rutting of soils generally increases the rolling resistance of tyres [5] leading to decrease in efficiency. The soil rut depth is also a useful measure, due to its simplicity, of the amount of soil compaction [6]. The soil compaction can lead to soil degradation by enhancing harmful physical, chemical and biological processes, which may affect the energy used in crop production [7]. Anderson [8] also uses the rutting of terrains to assess the level of vehicle impact on the environment. Therefore the development of a sensor to automatically and accurately measure soil deformation would lead to better energy efficiency not only in the vehicles themselves but also the in the production of crops,

Numerous methods have been developed to measure road profiles. General Motors [9] used a displacement transducer to measure the relative distance between the road and vehicle and an inertial measurement system using an accelerometer to measure the body displacement. This system was used to measure the low wavelengths of a single track and highlights the need of the profiler to determine the absolute position of the vehicle body. Kern and Ferris [10] developed a 3 Dimensional (3D) version using a line scan laser measuring a 
lateral line across the road. The lateral lines are then pieced together to form a $3 \mathrm{D}$ surface, which requires accurate measurement of the laser sensor's orientation and position. This results in an expensive system using an expensive displacement transducer and a highly accurate INS. More cost effective systems replace the displacement transducer with cheaper alternatives such as cameras [11] and or a road following wheel [12]. However, all these systems still require the use of an expensive INS system to obtain orientation and position of the sensor.

This paper proposes an inexpensive road profiling system where a 3D road profile is measured using photogrammetric methods based on two cameras. The cameras not only provide a full 3D surface with lateral, longitudinal and vertical coordinates with every measurement taken but also obtain a colour bitmap of the surface. The images are captured such that consecutive images overlap. The overlap allows the joining of 3D surfaces using both the colour bitmap and 3D surfaces. This method of joining negates the use of an accurate and expensive INS system, however, over longer distances accumulated errors may be large and the use of a slow sampling and inexpensive INS system is advised to remove drift.

\section{Texture Through Disparity (Stereovision)}

The method used to obtain depth is very similar to one of the ways a human captures depth though the use of two eyes. The technique is also commonly used in robots to capture depth. Stereovison (see Figure 1) works by determining the horizontal coordinate difference of an object $P$ projected onto the left and right imaging planes of the stereovision cameras to obtain points $P_{l}$ and $P_{r}$ lying at pixel locations $I_{l}(x, y)$ and $I_{r}(x, y)$ respectively. This difference in horizontal coordinate position between the point projected onto the two imaging planes, $I_{l}(x)-I_{r}(x)$, is commonly referred to as disparity. The disparity of a point can be mathematically shown to be inversely proportional to the depth $z$ of the point $P$. It can easily be shown that if the geometry of the system, the orientation and translational position between the two cameras, as well as the camera intrinsic calibration parameters are known then the $(x, y, z)$ coordinate position of a point $P$ can be mathematically determined [13]. The left pixel location and disparityof a point is mapped to the $(x, y, z)$ location of that point using a transformation matrix. The transformation matrixes as well as all calibration values are obtained using a calibration process shown in [14]. The main object of stereovision algorithms are the disparity mapping between the two images. There are various algorithms used to determine the disparity from two images. One of 
the most basic and fasted algorithms is the so called block matching algorithm [15]. The block matching algorithm computes stereo correspondence (mapping of object in one image to another image) by means of a

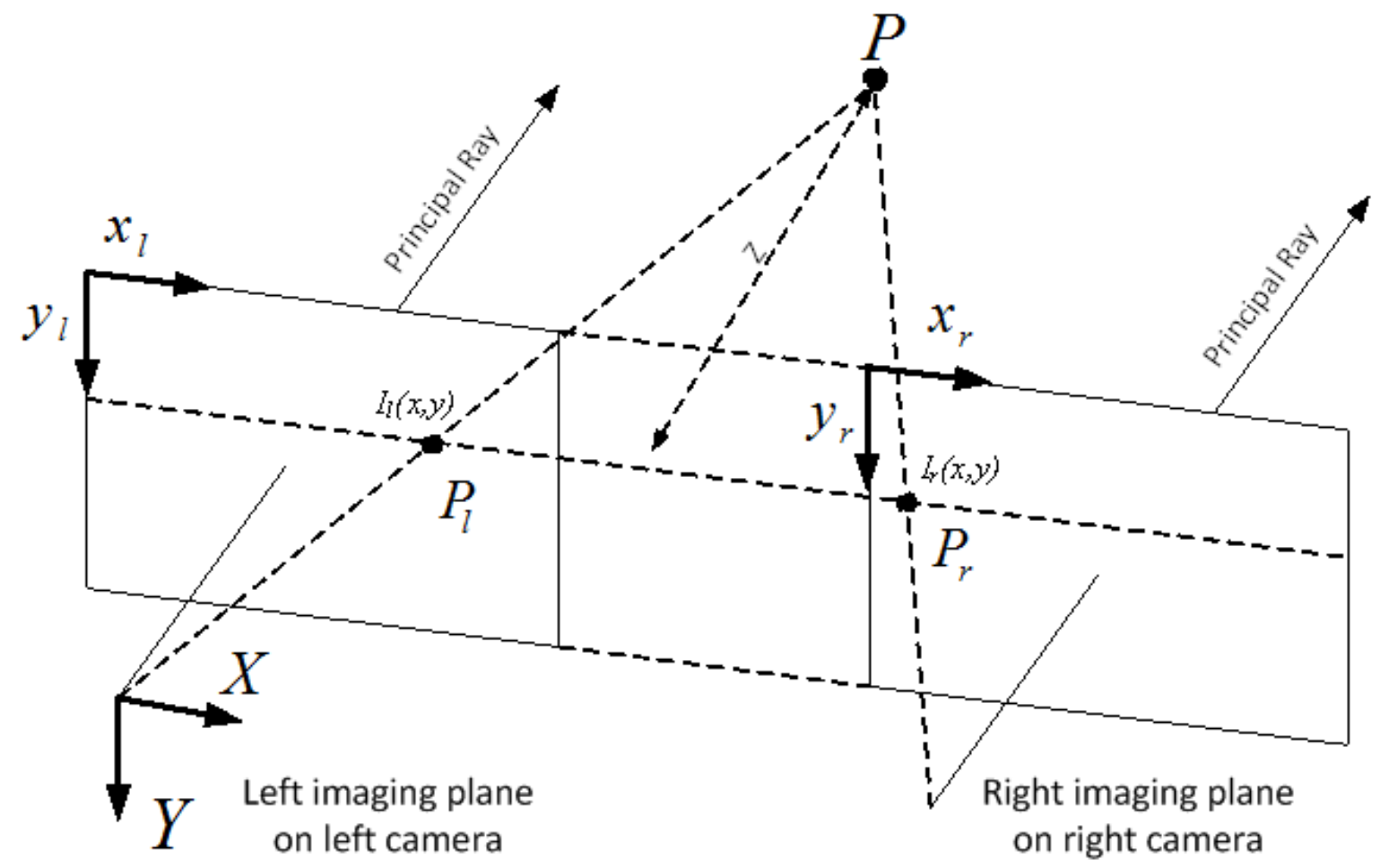

Figure 1 Stereo vision methodology

suitable metric. The most common metric is the Sum of Absolute Differences (SAD) of a moving window (NxN window). To simplify the searching procedure the camera calibration is also used to remap the camera images onto new imaging planes of which the projected points of a point $P$ in one image will lie on the exact same horizontal line as the other image, this line is commonly referred to as an Epipolar line. Therefore the vertical position difference between the two projected points is zero i.e. $I_{l}(y)-I_{r}(y)=0$.

Thus, the block matching algorithm performs line searches instead of an exhaustive global search over the whole image. The SAD metric used to detect correspondence is defined as:

$$
S A D=\sum_{i=-N / 2}^{N / 2} \sum_{j=-N / 2}^{N / 2}\left|I_{l}(x+i, y+j)-I_{r}(x+i+d, y+j)\right|
$$


where $N$ is the window size and $d$ is the disparity. The outline of the algorithm is to take every pixel in the left image $I_{l}(x, y)$ and then perform a line search on the right image to obtain the same projected point on the that image $I_{r}(x, y)$. The location corresponds to where the SAD metric is a minimum. After the disparity of each pixel has been established the real world $(x, y, z)$ location of that point relative to the cameras can be obtained. In some algorithms additional heuristic methods are applied to improve correspondence performance and speed.

This methodology requires that the surface has some arbitrary features which creates luminosity variations across the images that can be used for correspondence, thus it may be very difficult to apply this technique to monotone surfaces. However, most roads have arbitrary features such as grooves, stones and other discolourations which can be used for correspondence. More complex techniques with multiple variations, as well as pre and post filtering to improve disparity estimation are also available. However, the block matching technique is chosen due to its simplicity and fast solving time while still providing an accurate estimate of disparity. The Open Computer Vision (OpenCV) library [15] is used for calibration and stereo correspondence or disparity estimation.

The 3D coordinate of a pixel can be obtained using the pixel's $\mathrm{x}$, y coordinate and the disparity of the pixel. This is obtained using a transformation matrix which is obtained in a calibration process which effectively contains the orientations and translation between the cameras as well as the focal lengths of the cameras. This results in each pixel providing a 3D coordinate. Using two 640x480 resolution cameras a resolution of up to $640 \times 480$ can be obtained depending on the size of the region in view in both images. On average the region obtained contains 400x400 3D points. The spatial resolution between points depends on the focal lengths, resolution of the cameras as well as the distance from the surface to the cameras. The larger the area viewed the larger the spatial distance. The increase in viewing area however comes at a general loss of accuracy. This however can be compensated for by using higher resolution cameras. Higher resolution cameras come not only at a slightly higher monetary cost but a more substantially higher computational cost as well. Thus, when measuring depth with stereographic cameras, compromises between area, resolution and computational cost need to be considered. The cameras were setup so that the region captured was $250 \times 250 \mathrm{~mm}$ with a spatial distance between points of about $0.6 \mathrm{~mm}$. This region is large enough to encompass the contact patch of most SUV's and off-road vehicles. 


\section{Compilation of Surfaces}

In most 3D profilometers a line scan of the surface is obtained and joined using an INS system. However, with the method presented here every sample taken with the cameras creates a 3D surface instead of a 3D line. Thus, these surfaces and can be combined by simply having an overlap between consecutively sampled surfaces. The process of joining overlapping 3D surfaces is called registration. Three different registration techniques are applied to the individual surfaces to create a larger 3D surface.

The first technique uses 2D features obtained from the raw colour map of the camera images using Digital Image Correlation (DIC) techniques. DIC is a method whereby optical methods are used to track changes in an image. The methods can be used to obtain a variety of measurements ranging from displacements and velocities of particles to the strain of an object in an image [17]. There are numerous methods which can be used to perform DIC, however the underlying methodology remains the same. The techniques attempt to match regions in one image to regions in another, effectively tracking these regions across images, thus obtaining correspondences over the two images. The DIC technique is applied to consecutive images obtained from only the left camera. The left image is used since the disparity of the scene is obtained relative to this view. Thus, it is easy to obtain the $3 \mathrm{D}$ coordinates of pixels from this view.

To track a subset of regions from the image, first requires the identification of unique key points in both images. These key points are locations which should be unique i.e. easily differentiable from other key points, and would therefore be easy to identify in other images. Features which uniquely identify these regions are then extracted from the key points, effectively providing each key point with a fingerprint. The method of obtaining key points and the type of features extracted often makes up the main difference between DIC feature matching algorithms. Two robust feature descriptors often used are Scale-Invariant Feature Transform (SIFT) [18] and Speeded Up Robust Features (SURF) [19].

In this paper the SIFT feature descriptor is used. SIFT key points are determined by local minima and maxima from the result of applying difference of Gaussians to the image. This is performed on numerous scaled and blurred images from the same scene to obtain regions with high contrast that are scale invariant. An orientation is also assigned to each key point based on the direction of the local image gradient to obtain invariance to rotation. After key points have been assigned features are extracted around this region. The features are the magnitude and direction of the image gradient in a window of $4 \times 4$, where each grid point 
describes the magnitude of the gradient in 8 directions. In total 128 features are extracted per key point which creates a feature vector. The feature vector is normalized to obtain invariance to illumination.

An attempt is then made to match the key points from the two separate images therefore tracking the key points in from one image to another. This is performed by using a suitable distance metric between features, usually the Euclidean metric. The match of a key point in image $I_{t}$ is then obtained by determining the distances between key points in image $I_{t+1}$ either using an exhaustive brute force method or a random sampling method. The key point with the smallest distance is taken as the matching key point.

Once a set of 2D image correspondences between two images are obtained, with a relative change in motion between them, the 3D coordinates of these correspondences can be obtained as well. This yields several 3D points in space which have undergone rigid motion, rotation and translation, between two views of which the position is known in both views. The relative translation and rotation can then be determined provided at least three point correspondences have been obtained [20]. The problem being solved is the rotation matrix $\boldsymbol{R}$ and translation vector $\boldsymbol{t}$ in:

$$
P_{2}=R P_{1}+t
$$

Where the $N$ 3D points set $\boldsymbol{P}_{\mathbf{1}}$ undergoes rigid motion to yield the points set $\boldsymbol{P}_{\mathbf{2}}$. The method starts of by finding the centres of the $\boldsymbol{P}_{\mathbf{1}}$ and $\boldsymbol{P}_{\mathbf{2}}$ :

$$
\overline{\boldsymbol{P}_{\mathbf{1}}}=\frac{1}{N} \sum_{i=1}^{N} \boldsymbol{P}_{\mathbf{1}}^{i}
$$

where $\boldsymbol{P}_{\mathbf{1}}^{\boldsymbol{i}}$ is the $i^{\text {th }}$ 3D point correspondence. Next the covariance matrix between points set $\boldsymbol{P}_{\mathbf{1}}$ and $\boldsymbol{P}_{\mathbf{2}}$ are determined as:

$$
\boldsymbol{\Sigma}=\sum_{i=1}^{N}\left(\boldsymbol{P}_{\mathbf{1}}^{\boldsymbol{i}}-\overline{\boldsymbol{P}_{\mathbf{1}}}\right)\left(\boldsymbol{P}_{\mathbf{2}}^{\boldsymbol{i}}-\overline{\boldsymbol{P}_{2}}\right)^{T}
$$

The rotation matrix $\boldsymbol{R}$ is obtained from the Singular Value Decomposition (SVD) of the covariance matrix:

$$
\begin{aligned}
& \boldsymbol{\Sigma}=\boldsymbol{U} \boldsymbol{S} \boldsymbol{V}^{T} \\
& \boldsymbol{R}=\boldsymbol{V} \boldsymbol{U}^{T}
\end{aligned}
$$


If the determinate of $\boldsymbol{R}$ is negative the solution corresponds to an inflection. The rotation matrix is then recalculated as:

$$
\boldsymbol{R}=\boldsymbol{V}\left[\begin{array}{ccc}
1 & 0 & 0 \\
0 & 1 & 0 \\
0 & 0 & -1
\end{array}\right] \boldsymbol{U}^{T}
$$

The translation vector is the obtained from:

$$
t=\overline{P_{2}}-R \overline{P_{1}}
$$

While the above equation requires at least 3 point correspondences, the method will calculate the rotation and translation in a least mean square sense for any point sets with $N$ correspondences. Thus, several point correspondences $(\approx 50)$ are used. However, to prevent any outliers from influencing the calculation of the rotation and translation the RANdom SAmple Consesus (RANSAC) algorithm [21] is used to remove outliers. The RANSAC algorithm is an iterative procedure to estimate the parameters of a model from a set of data which is corrupted by outliers. The synopsis of the method is to randomly sample a minimum set of data points for which to develop the model, in this case 3 correspondences are used to obtain the translation vector and rotation matrix. The model is derived from this minimal set and each remaining data point is tested against the model using a suitable metric, in this case $\epsilon=\left\|\boldsymbol{P}_{2}^{i}-\left(\boldsymbol{R} \boldsymbol{P}_{1}^{i}+\boldsymbol{t}\right)\right\|$. If the error of the metric is within some suitable error the data point is considered to agree with the model and counted as an inlier. The process of sampling a minimum set of data points and developing a model continues for a suitable amount of iterations. Upon completion the model with the most inliers is returned along with the set of inliers. The rotation and translation is then determined in a least square sense using all of the inliers of the best model.

This then yields the relative orientation and translation between two consecutive frames. The rotation and translation is calculated at each sample with the point correspondences relative to the camera. Thus, each calculated rotation and translation presents the motion which occurred from sample one to sample two, relative to sample one. The rotation and translation matrices are combined into one transformation matrix in homogeneous coordinates as:

$$
\boldsymbol{T}_{\text {local }}=\left[\begin{array}{cccc}
R_{11} & R_{12} & R_{13} & t_{x} \\
R_{21} & R_{22} & R_{23} & t_{y} \\
R_{31} & R_{32} & R_{33} & t_{z} \\
0 & 0 & 0 & 1
\end{array}\right]
$$


where $R_{i j}$ represents the component from the $i^{\text {th }}$ row and $j^{\text {th }}$ column in the rotation matrix $\boldsymbol{R}$ and $t_{x}$ is the translation in the $x$-direction. The transformation is then performed as:

$$
\left[\begin{array}{c}
P_{2 x}^{i} \\
P_{2 y}^{i} \\
P_{2 z}^{i} \\
1
\end{array}\right]=\boldsymbol{T}\left[\begin{array}{c}
P_{1 x}^{i} \\
P_{1 y}^{i} \\
P_{1 z}^{i} \\
1
\end{array}\right]
$$

where the point correspondence $\boldsymbol{P}_{\mathbf{1}}^{\boldsymbol{i}}$ is appended by 1 to create a homogeneous coordinate. This multiplication performs both the rotation and translation in one single matrix multiplication. It also allows the easy accumulations of rotations and translations from the individual samples to calculate the global position and orientation of each sample's surface as:

$$
T_{\text {total }}=T_{\text {total }} T_{\text {local }}
$$

This method is computationally fast compared to the other two registration techniques used, which work directly on the 3D point clouds produced at each sample. As such this technique is also used to determine when the other registration techniques are to be used. This is accomplished by determining the distance travelled since the last time a 3D point cloud registration technique was used. Once a certain distance has been travelled which would assure a certain percentage in overlap, the system triggers the use of an additional registration technique.

One of the additional registration techniques used is that of Iterative Closest Point (ICP). The ICP algorithm works by first obtaining the closest matches of each point in the original cloud (sample one) to the transformed point cloud (sample two). This step is by far the most time consuming and modifications to the ICP algorithm are generally directed at speeding up the matching. The ICP algorithm proposed by Besl and McKay [22] calculates the distance from each point in the original cloud to all points in the transformed cloud to obtain the closest point. This process is time consuming as it performs an exhaustive search. More efficient variations are also available [23]. The algorithm then obtains a rigid transformation which reduces the distances between the matches. The process finds a local minimum and thus requires several iterations before convergence is reached. The ICP algorithm used is implemented in the Open Point Cloud Library [24] The transformation matrix return by ICP is then used to update the total transformation matrix as:

$$
T_{\text {total }}=T_{I C P} T_{t o t a l}
$$


The ICP algorithm works best if the point clouds are initially aligned to some degree. This initial alignment is performed by the first registration algorithm, however, if the point cloud cannot find matches an additional registration technique is used to perform the initial alignment.

This additional registration technique used is very similar in approach to the first, with the exceptions that both key points and features are obtained from the spatial domain, i.e. from the 3D point cloud, rather than from the colourmap of the raw images. Tombari et al [25] evaluated the performance of various 3D key point detectors and found that Intrinsic Shape Signatures (ISS) [26] key points provide good performance while being particularly efficient. The algorithm works by determining the covariance matrix of points surrounding a proposed key point. The eigenvalues of the covariance matrix is used to determine the saliency of the key point. If the ratios of the successive eigenvalues are below a certain threshold then the point is kept. The rationale is to avoid detecting key points at points which have a similar spread about the principal directions where a repeatable canonical reference cannot be established. The saliencies of the remaining points are determined by the value of the smallest eigenvalue. This produces a method which is efficient in finding reasonable key points. After key points have been identified, features describing the region need to be determined. For this Point Feature Histograms are often used [27]. Wahl et al [28] describes four features that can be created from the distance vector between two points and their surface normal. The process is then to obtain these features between all points within a certain radius of the key point. Each feature is then divided into a histogram with a certain number of bins. Each bin contains the amount of points within the region that falls within the bin range. The feature vector thus contains 4 histograms of $n$ bins. The feature vectors can then be mapped using the Sample Consensus - Initial Alignment (SAC-IA) [29] method. In this method a subset of samples are drawn from the first image and a list of possible matches are obtained in the second image using a suitable metric, such as Mahalanobis or Euclidean distance. A correspondence is chosen at random from the list and the rigid transformation is computed from the correspondences. The quality of the transform is then determined, this process is repeated and the best transformation is returned. This transformation is then applied to the point cloud after which another iteration of ICP is used to refine the registration.

\section{Interpolation}

With the registration techniques applied multiple point cloud surfaces are obtained which overlap by about $50 \%$. These point clouds require interpolation or subsampling to obtain a final point cloud which averages over all point cloud surfaces. Detwieler and Ferris [30] conducted a study on interpolation methods for three- 
dimensional terrain surfaces. They concluded that the Inverse Distance to a Power method was the recommended method when fine resolution is required to describe localized roughness and that Kriging is ideal for identifying global trends of the surface.

\subsection{Inverse Distance to a Power Method}

The Inverse Distance to a Power method is a weighted average interpolator. With this method data is weighted according to their distance from the node under consideration, with points closer receiving a higher weighting than those further away. The weighting function can be also be altered using a weighting power, this power controls how quickly the weighting changes with distance. The higher the power the less impact the points further away from the node has on the value of the node.

\subsection{Kriging}

Kriging is a geostatistical estimator method. It follows in principle the same methodology as the Inverse Distance to a Power method whereby the predicted value is the weighted average of surrounding points. It differs in how weights are assigned to surrounding points. While the Inverse Distance to a Power method is a deterministic interpolation method where weights are based directly on the values of surrounding points, in Kriging a statistical model is fitted to surrounding points. Kriging thus assumes that the distance and direction of points reflects a spatial correlation that can describe variations in the surface. Kriging is therefore a useful interpolation tool which can capture global trends in data such as long wavelength sinusoidal undulations and other repeated patterns. Kriging is however a very computationally expensive algorithm which takes an order of magnitude longer than the Inverse Distance to a Power method to solve.

Since most off- road terrains can be considered random, with the exception of washboard roads and bricked roads (Belgian paving), the Inverse Distance to a Power Method is used to perform the interpolation. The algorithm is also much less computationally expensive than Kriging thus reducing the interpolation time. The Inverse Distance to a Power Method equation is given as:

$$
Z_{\text {interp }}(\overline{\boldsymbol{x}})=\frac{\sum_{i=1}^{N} w_{i} z_{i}(\boldsymbol{x})}{\sum_{i=1}^{N} w_{i}}
$$

where $w_{i}$ is the weighting given as:

$$
w_{i}=\frac{1}{d(\overline{\boldsymbol{x}}, \boldsymbol{x})^{P}}
$$


where $d(\overline{\boldsymbol{x}}, \boldsymbol{x})$ is the Euclidean distance between the point being interpolated $(\overline{\boldsymbol{x}})$ and the point from the original point cloud (x). The domain of $N$ points is taken as all points which lie within a certain radius from the interpolation point. Most often the points are obtained from an exhaustive search over all points to obtain those which lie within this radius. However, since the 3D surface obtained from the stereovision system has a certain structure, the domain of points used for interpolation can be obtained in a much faster way. The underlying structure of the surface, which is made up of a 3 matrices $[\mathbf{X ~ Y ~ Z ~} \mathbf{Z}$, is that the longitudinal $(\mathbf{X})$ and lateral distances (Y) when mapped according to the row (i) and column (j) indices form a plane, as shown in Figure 2.
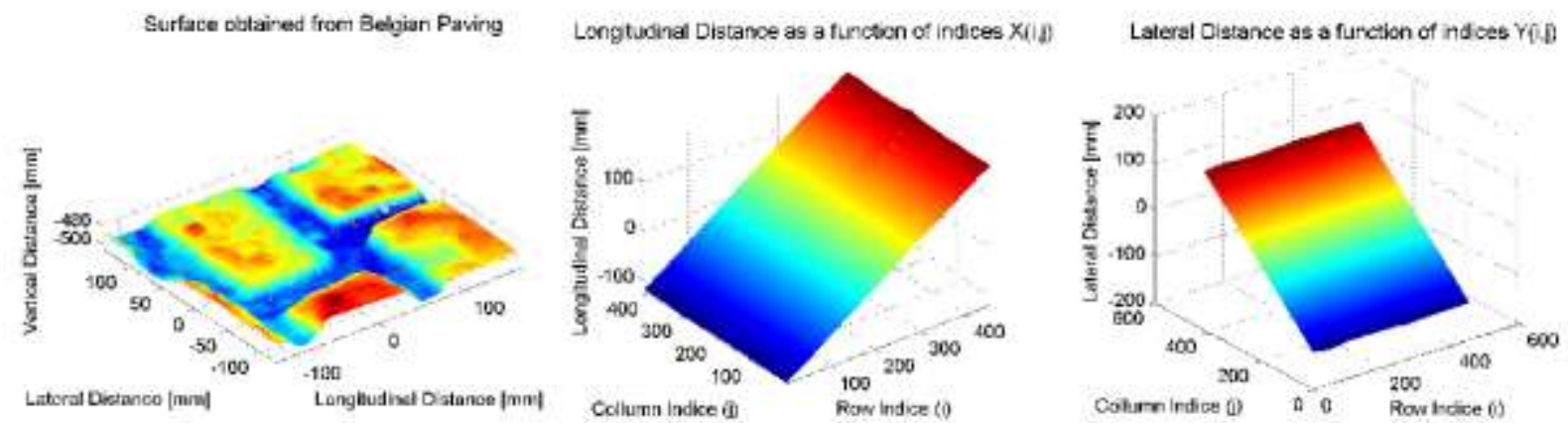

Figure 2 Measured surface. Longitudinal distance value as a function of index. Lateral distance value as a function of index.

This results in a fairly consistent increase in longitudinal and lateral distances as the row and column indices are incremented. As a result of this a much faster interpolation strategy is proposed which does not perform an exhaustive search over all points in the point cloud. The process starts by determining the bounds of each surface $\left[\begin{array}{llll}x_{\min } & y_{\min } & x_{\max } & y_{\max }\end{array}\right]$. Next the incremental increase in longitudinal and lateral distance, as the row (i) and column (j) indices are incremented, is determined. These values are given as $\left[\begin{array}{llll}x_{\text {step } \_} i & x_{\text {step } \_j} & y_{\text {step } \_} i & y_{\text {step } \_} j\end{array}\right]$, where $x_{\text {step } \_i}$ is the increase in longitudinal distance as an increase in the row index (i). Therefore, each longitudinal and lateral value as a function of the matrix indices can be described as:

$$
x_{i, j} \approx x_{0,0}+i x_{\text {step }_{-} i}+j x_{\text {step }}
$$




$$
y_{i, j} \approx y_{0,0}+i y_{\text {step }_{-} i}+j y_{\text {step }_{j}}
$$

Where $x_{i, j}$ is the longitudinal position of point $(i, j)$. If the equations are solved for in terms of indices $(i, j)$, one obtains:

$$
\begin{aligned}
& j \approx \frac{y_{0,0}-\frac{x_{0,0}}{x_{\text {step }}} y_{\text {step } \_} i}{y_{\text {step } \_}-\frac{x_{\text {step }}}{x_{\text {step }}} y_{\text {step } \_} i} \\
& i \approx \frac{x_{0,0}-j x_{\text {step } \_j}}{x_{\text {step } i} i}
\end{aligned}
$$

This allows one to obtain the indices $(i, j)$ given the longitudinal and lateral position $(x, y)$ and knowing the start position $\left(x_{0,0}, y_{0,0}\right)$. Since this is only an approximation, the process can be repeated a few times $(2-3$ times) by simply changing (17) and (18) as:

$$
\begin{aligned}
\Delta j \approx \frac{\Delta y-\frac{\Delta x}{x_{s t e p_{i}}} y_{\text {step } i} i}{y_{s t e p_{-} j}-\frac{x_{s t e p_{j}}}{x_{\text {step }}} y_{\text {step } p_{-} i}} \\
\Delta i \approx \frac{\Delta x-j x_{\text {step } j} j}{x_{\text {step } i} i}
\end{aligned}
$$

This should then converge to the region in the surface where the point being interpolated should lie. The interpolation is then performed with radii in the $\Delta i$ and $\Delta j$ direction obtained from (19) and (20) by substituting $\Delta x$ and $\Delta y$ by the search radius $R$. While the interpolation region is not strictly circular but rather rectangular this has a very small effect on the interpolated value especially with a large search radius $R$. Thus, the interpolation process can be summarized as:

1. Find all surfaces in which the point $(x, y)$ lie using the ranges of the surfaces $\left[\begin{array}{llll}x_{\min } & y_{\min } & x_{\max } & y_{\max }\end{array}\right]$

2. Find the $(i, j)$ indices where the point $(x, y)$ lies within a surface using $(17)-(20)$ 
3. Find the search region in terms of indices using (19) and (20)

4. Interpolate over all surfaces using the Inverse Distance to a Power Method

This process works as long as the point $(x, y)$ lies within at least one surface. If the point is found to lie outside all surfaces an extrapolation procedure is used. The extrapolation procedure assigns the value to the point of its nearest neighbour. To find the nearest neighbour the surfaces are ranked in terms of distance from the point using the point $\left(x_{0,0}, y_{0,0}\right)$ of the surfaces. Starting at the closest surface a boundary search is performed in that surface and the closest point retained, a boundary search is then computed for the next closest surface. This process is repeated until the closest boundary point in the current surface is further away than the closest boundary point found in the previous surface, thus halting the process. While the interpolation process can be considered to be fairly fast the extrapolation process is still regarded as slow in comparison.

\section{Experimental Results}

\subsection{Laboratory calibration tests}

The accuracy of the stereovision, registration and interpolation procedures are evaluated using a small calibration surface. Smith and Ferris [31] suggest that a calibration surface should contain contours with sharpedged high-bandwidth events. The surface thus consists not only of round contours but also contours with sudden changes in height or sharp edges. The surface is sprayed with a speckle pattern to aid the stereovison system in determining the depth. The calibration surface along with the ground truth of the surface is shown in Figure 3.
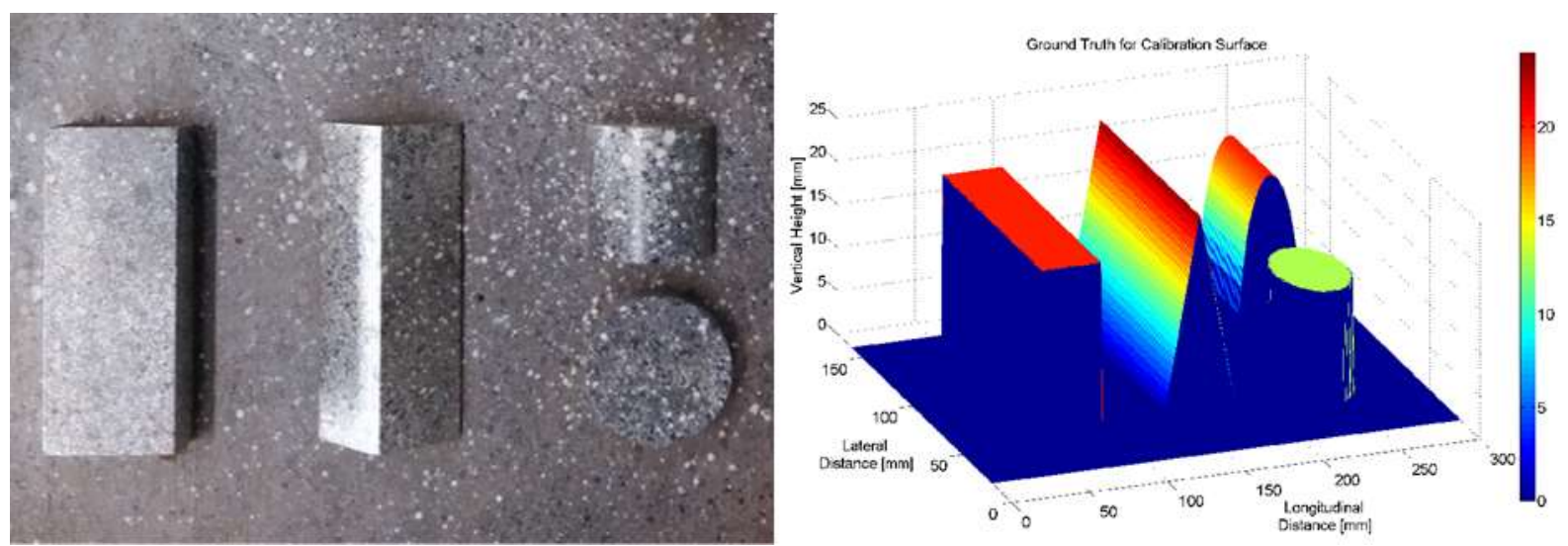

Figure 3 Calibration surface a) photo b) ground truth. 

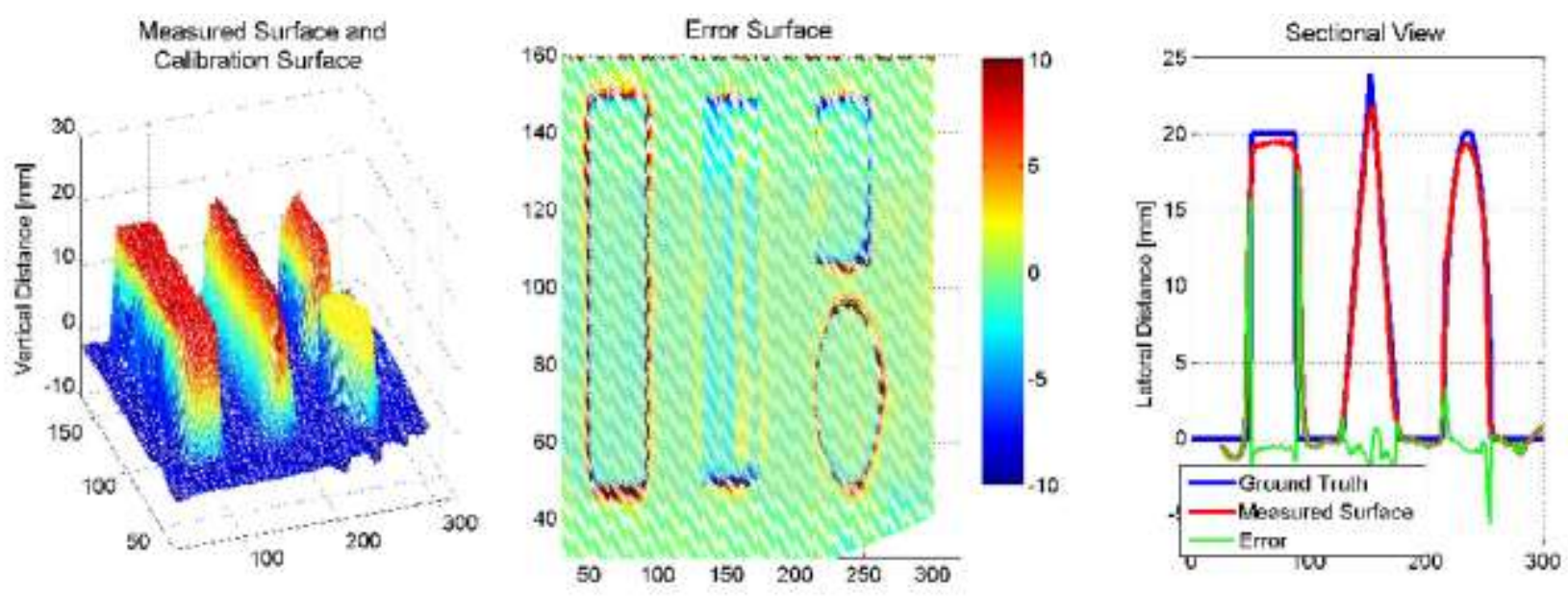

Figure 4 Final interpolated surface. Error surface of measured surface. Sectional view.

The cameras are Point Grey Chameleon USB 2 cameras with maximum resolution of 1296 x 964 and a global shutter. The lens is a Fujinon $6 \mathrm{~mm}$ focal length c-mount lens. The camera resolution is set fairly low at 640x480 to speed up computation. The surface is measured by moving the stereovision system across the system by hand. The final surface is a $1 \mathrm{~mm}$ spaced interpolated surface obtained from 15 point cloud surfaces which are joined together. The final measured surface is shown in the first plot of Figure 4 . The second plot shows the vertical distance error surface. The error is therefore merely the difference in vertical distance, this error metric is fairly conservative as compared to an error distance which measures the distance to the nearest ground truth point. This plot indicates that large errors consist at the edges of objects which have sharp edges. This is partly due to the metric used as well as that these regions may never have been seen by both cameras as the cameras were still pointed down vertically. The problem of hidden regions is a problem with most measurement systems especially optically based systems. It can generally be corrected by viewing the regions at different angles which would enable the system to then measure these regions. While the system easily allows this to be performed, this method is however not considered in this paper. In this paper most measurements are made vertical to the surface. Thus, at these sharp edges the surface is not measured and therefore these regions are interpolated causing large errors. The RMS error over the whole error surface is $2.4 m m$, however this is mostly dominated by the larger errors at sharp edges. The last plot shows a sectional view of the profiles as well as the error surface. The plot clearly shows the error at the sharp edges. However, at the smoother areas the errors are much smaller staying mostly below $1 \mathrm{~mm}$. This indicates that the system slightly smooths sharp edges. The smoothing is a result of both the disparity mapping which slightly smooths regions, based on the window 
size, as well as the registration technique which may cause slight smoothing. In vehicle dynamics applications this is not of significant concern since the tyre itself smooths sharp edges to a greater extent than the stereovision system. The measurement system accuracy can still be improved with higher resolution settings as well as changing the camera geometry, this is however not performed in this study. The system is deemed to have satisfactory result for the purpose of terrain measurements.

\subsection{Real road tests}

The stereovision system was experimentally tested on real road surfaces on the suspension track of the Gerotek Testing Facilities [32]. The system was attached to another road profiler as described by [12]. The system consists of a beam with three wheels and multiple road following arms attached to potentiometers, as the arms move over the surface the change in angle is measured which is related to the height of the arm. This allows the height to be calculated at each arm relative to the beam. The system is also equipped with a Novatel SPAN-CPT GNSS+INS receiver [33] which allows the measured motion relative to the beam to be transformed into motion relative to a global reference. The system therefore allows the mapping of a terrain in global coordinates with a resolution of $40 \mathrm{~mm}$ lateral and $6 \mathrm{~mm}$ longitudinal. The road profiler is shown in Figure 5.
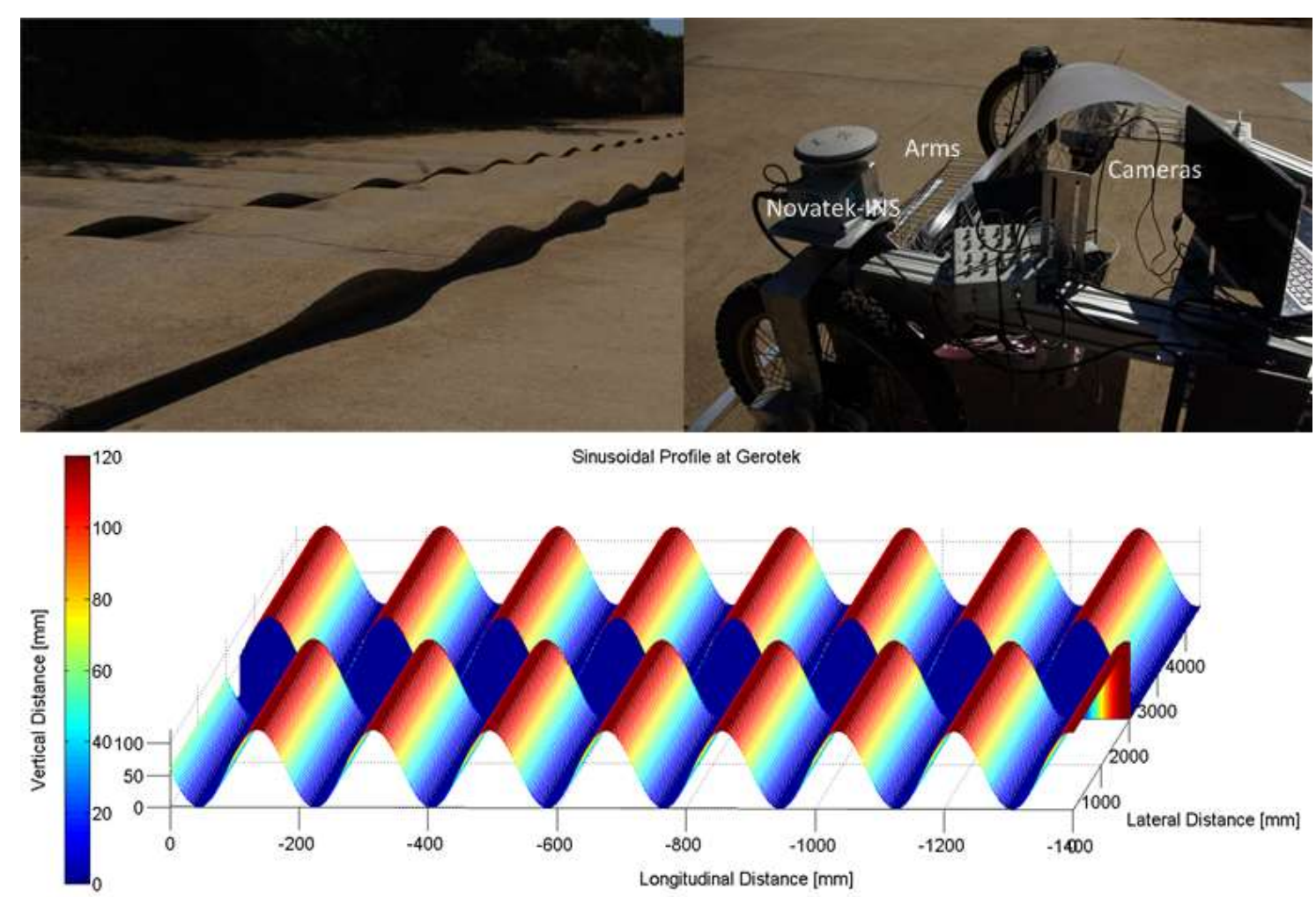

Figure 5 Sinusoidal profile. Experimental setup. 
The profiler with the camera system was tested over multiple surfaces which introduce various forms of excitations to the system. Due to the design of the arm based system it is referred to as the CanCan machine in this study. The experimental setup along with some of the road profiles used, such as sinusoidal profiles, is shown in Figure 5. At the height mounted the camera measured an individual surface of dimensions $260 \times 260 \mathrm{~mm}$ containing a grid of $400 \times 400$ points, with a spacing of about $0.65 \mathrm{~mm}$ between points.

\subsubsection{Flat Surface}

A flat $4 m$ long section was profiled first, the final cloud is interpolated from 38 individual surfaces. It was found that due to insufficient calibration that the measured surface contained drift. The insufficient calibration causes a curvature error in the individual surfaces captured. The combined surfaces thus gradually cause the final surface to drift. The drift is also not linear as the curvature error exponentially increases the vertical height drift. As a result the drift is removed by means of detrending. The detrending is achieved by fitting a 2 degree polynomial through the surface along the longitudinal distance. This polynomial, which captures the mean of the surface, is subtracted from the original surface. This yields a surface from which low frequency drift is removed without affecting the higher frequencies. Figure 6 shows the surface profile as well as the detrended surface, the detrended surface removes almost all drift from the surface. In order to evaluate the camera based profiler the camera measurements such as roll, pitch and vertical height are detrended as well using a single degree polynomial.

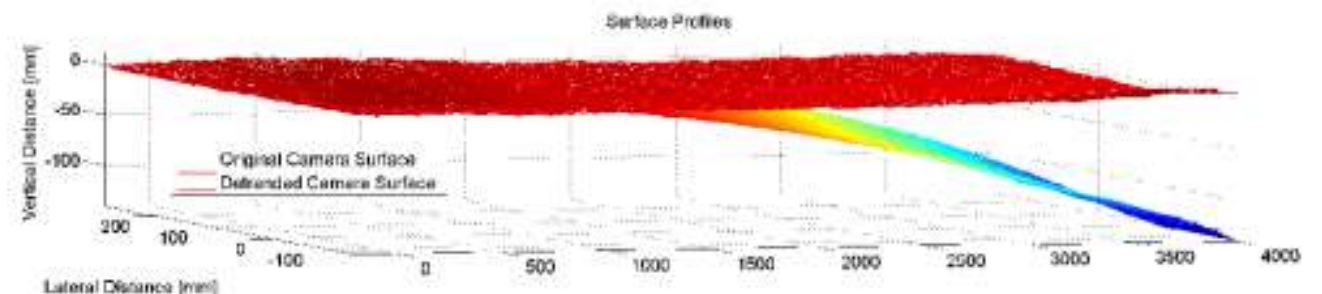

Luteral DGosancos jnes

Lavest Disterce [inm]

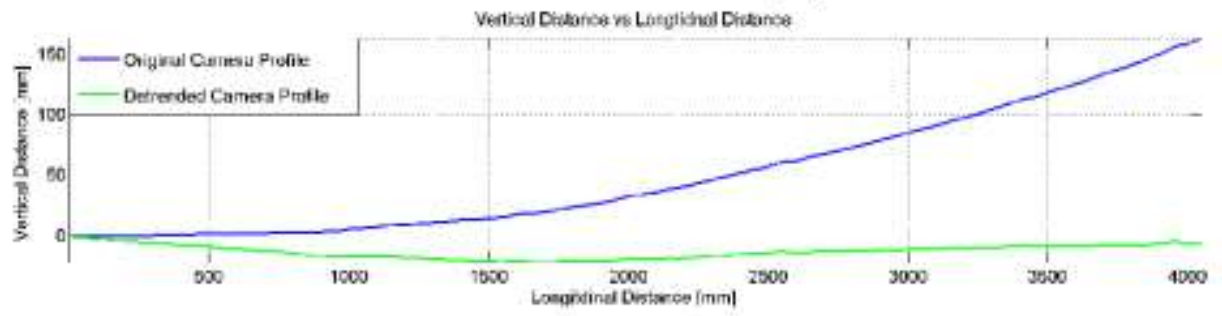

Figure 6 Measured surface and vertical displacement measuring a flat surface. 


\subsubsection{Roll Excitation}

The profilometer was excited in the roll direction by letting the left wheel follow a sinusoidal vertical motion while the two right wheels remained at a constant vertical height. The vertical profile followed by the left wheel is a $60 \mathrm{~mm}$ amplitude sinusoid with a $1800 \mathrm{~mm}$ wavelength, see Figure 5 (left and below). The Stereovision camera system thus experienced a roll motion while still profiling a flat surface. The roll and pitch angles experienced by the profilometer are used to evaluate the ability of the stereographic profiling system to remove the roll excitation from the profile.

The baseline measurements are obtained from the geometry of the problem, knowing the dimensions of the CanCan machine as well as the profile which each wheel followed. This allows for exact determination of the roll and pitch angles as well as vertical height of the sensors. In the case of the roll excitation the roll angle is additionally determined by road profiling lasers. This is achieved by placing two road profiling lasers a certain distance apart on the rear wheels. From the known and measured displacements the roll angle can be determined from basic trigonometry. This method is not used in the other excitations since the lasers could not measure a flat reference surface. The baselines are used to compare the measured angles from firstly the Novatek-INS which uses high accuracy fibre optic gyros to determine the roll, pitch and yaw angles and a GPS system combined with accelerations to determine the lateral, longitudinal and vertical position. Secondly the detrended measurements from the camera based system are used for comparison. The camera measurements are obtained by integrating the relative velocity measurements obtained between frames. Two data sets are used in this comparison. The difference in the data sets are mostly the accuracy obtained from the Novatek-INS. This is done in order to demonstrate the varying accuracy which can be expected from the GPS components of an INS system.

The first plot in Figure 7 shows the measurements obtained from the best data set. The figure shows that the Novatek-INS measures the roll and pitch angles perfectly, following the ideal and laser measurements very closely. The detrended camera measurements also compare well to that of the baseline measurements. The camera measurements have the correct amplitude and frequency, however showing slightly more noise than the Novatek-INS. The noise amplitude is comparable to most MicroElectroMechanical Systems (MEMS) based INS systems. This demonstrates that the system successfully measures the roll excitation. 

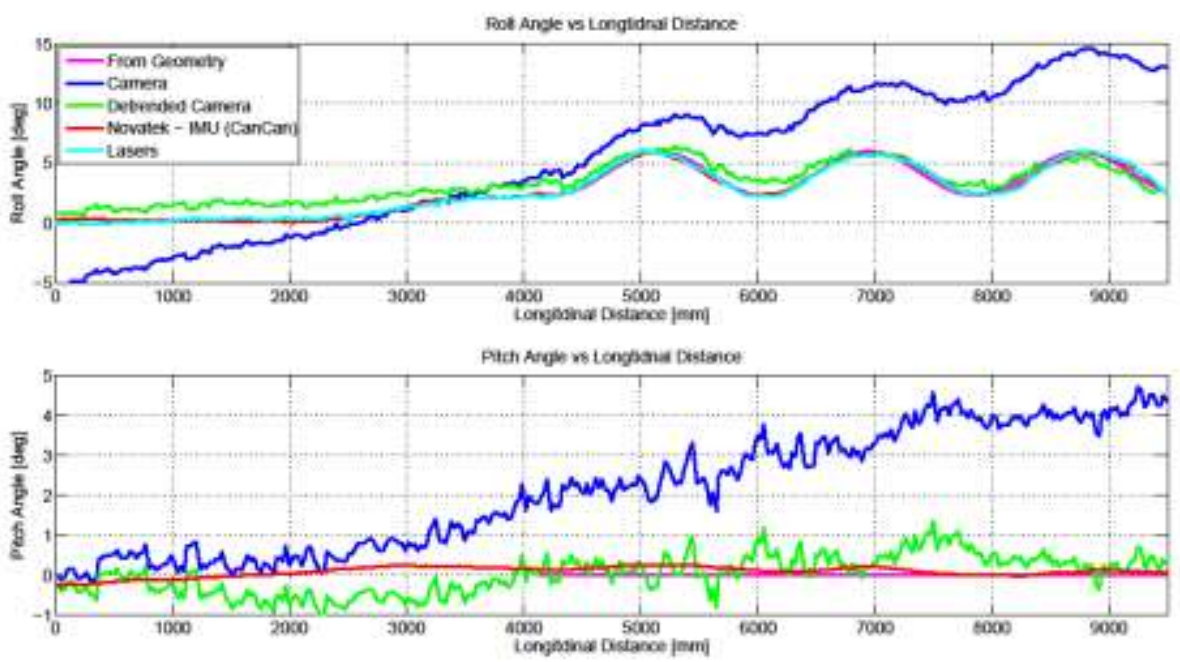

Figure 7 Measured roll and pitch angle with roll excitation, best INS measurement.
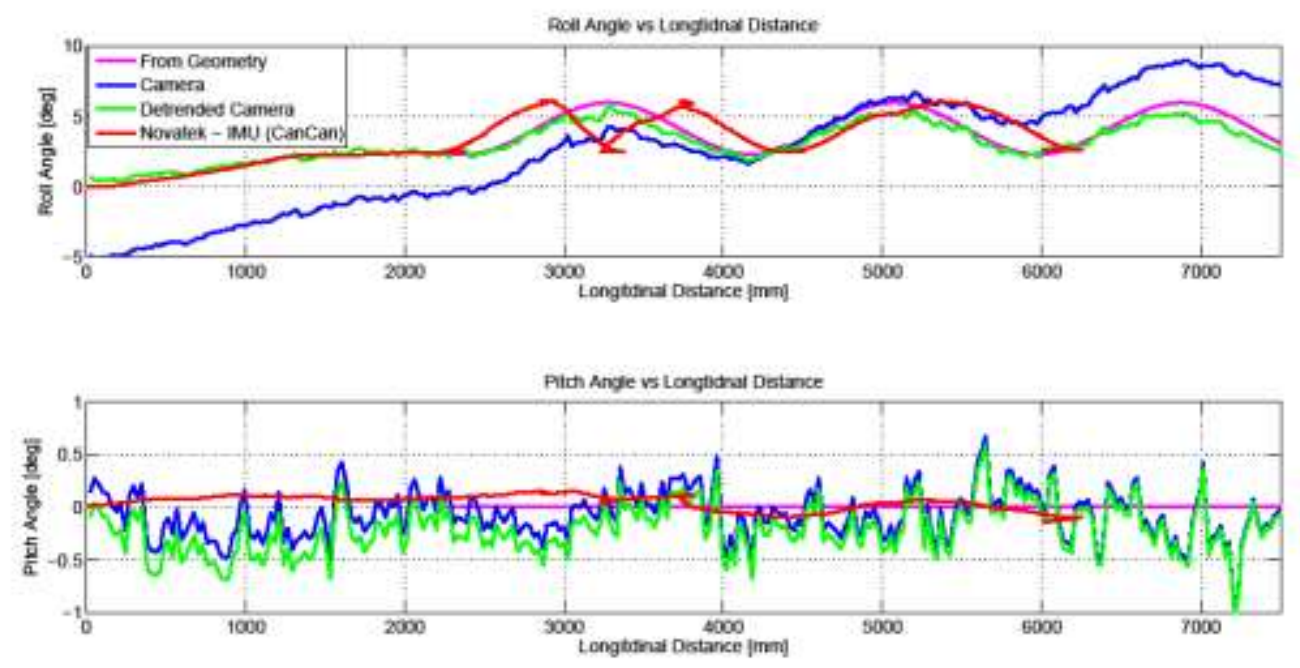

Figure 8 Measured roll and pitch angle with roll excitation, worst INS measurement

Figure 8 shows the same plots for the worst INS measurement. The plots indicate that while the INS system obtains the correct amplitudes of the angles the longitudinal displacement is incorrect. This is to be expected since the angles are obtained using high accuracy fibre optic gyroscopes. However, the longitudinal position is obtained primarily from GPS, with some filtering using accelerometers. GPS measurement accuracy is highly dependent on the satellite constellation and varies during the day. A jump in position can also occur when a satellite is lost or a new one is acquired. This results in GPS accuracy varying during testing causing repeatability problems. Even though the angles are measured accurately, most road profilers obtain the positions from the INS system and an error in the positions will drastically affect the final surface obtained. 
It is possible to improve the INS system accuracy significantly by utilising a GPS base station or Real Time Kinematic (RTK) GPS. The base station uses a stationary measurement point to improve and remove unnecessary jumps in GPS measurements. Use of such a system is expected to greatly improve the accuracy as well as consistency of the GPS. In comparison the results from the camera profiler are unaffected and remain consistent. This indicates that this system is very repeatable with few external factors affecting the accuracy. In Figure 9 the profiled surface with roll excitation is shown, the profile is as required fairly flat indicating almost complete removal of the roll excitation.

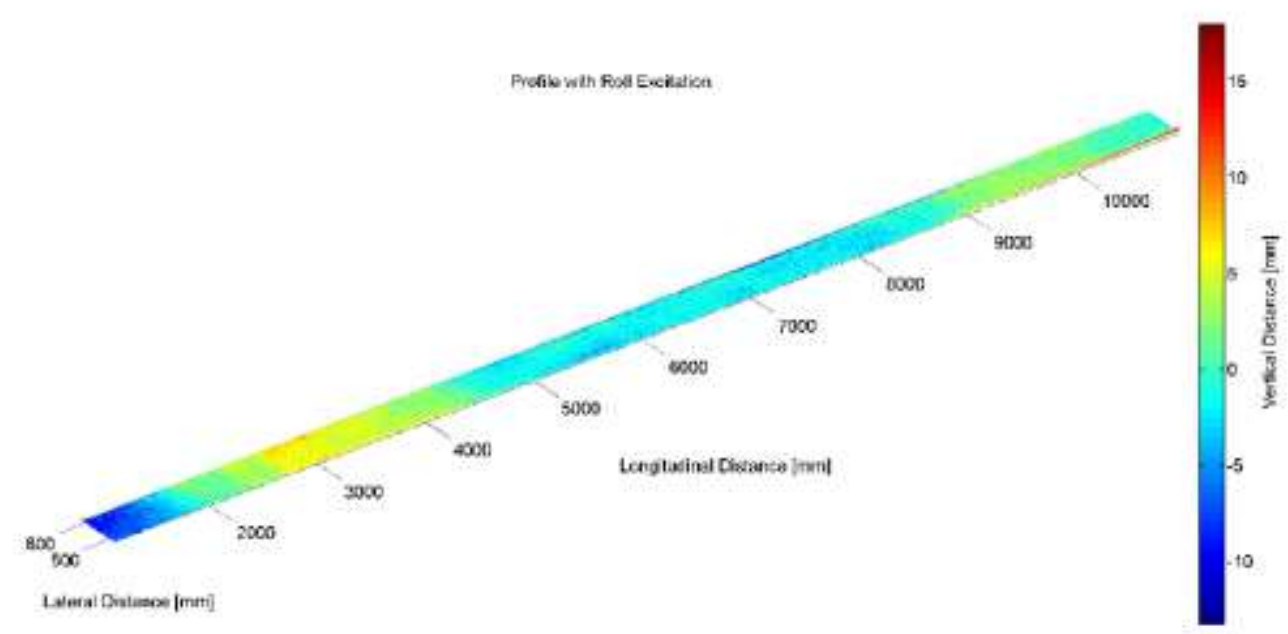

Figure 9 Measured flat surface with roll excitation.

\subsubsection{Pitch Excitation}

The system was next excited in the pitch direction. This was achieved by having all the wheels of the profiler move across a sinusoidal profile. This not only induces a pitch excitation but also a vertical motion of the measuring systems as well. The profiler also traverses the road while profiling the same sinusoidal profile. The plots in Figure 10 show that the pitch angles of both the camera and INS compare excellently to the baseline measurements. It should be noted that the ideal profile only starts around the $5000 \mathrm{~mm}$ mark, the section in front of this is an unknown profile. However, the camera and INS system still provide similar responses in this unknown region. The INS angle however has a slightly longer wavelength which results in the profiles being out of phase at the end of the test. This again shows the effect which can be induced by the small errors of the GPS system. While the camera measurements again contain more noise the measurements compare well to the baseline profile with similar amplitudes and the correct wavelength. The vertical height plot shows that the INS 
system also contains considerable drift, this is especially true in the vertical direction due to GPS having unsatisfactory vertical height accuracy. This can adversely affect the profiled terrain which relies heavily on all measurements from the INS system. Both systems measure similar amplitudes with the camera system measuring the correct wavelength compared to the INS system. Overall the camera vertical height measurement provides better results compared to the INS system which is noisier and contains random jumps.
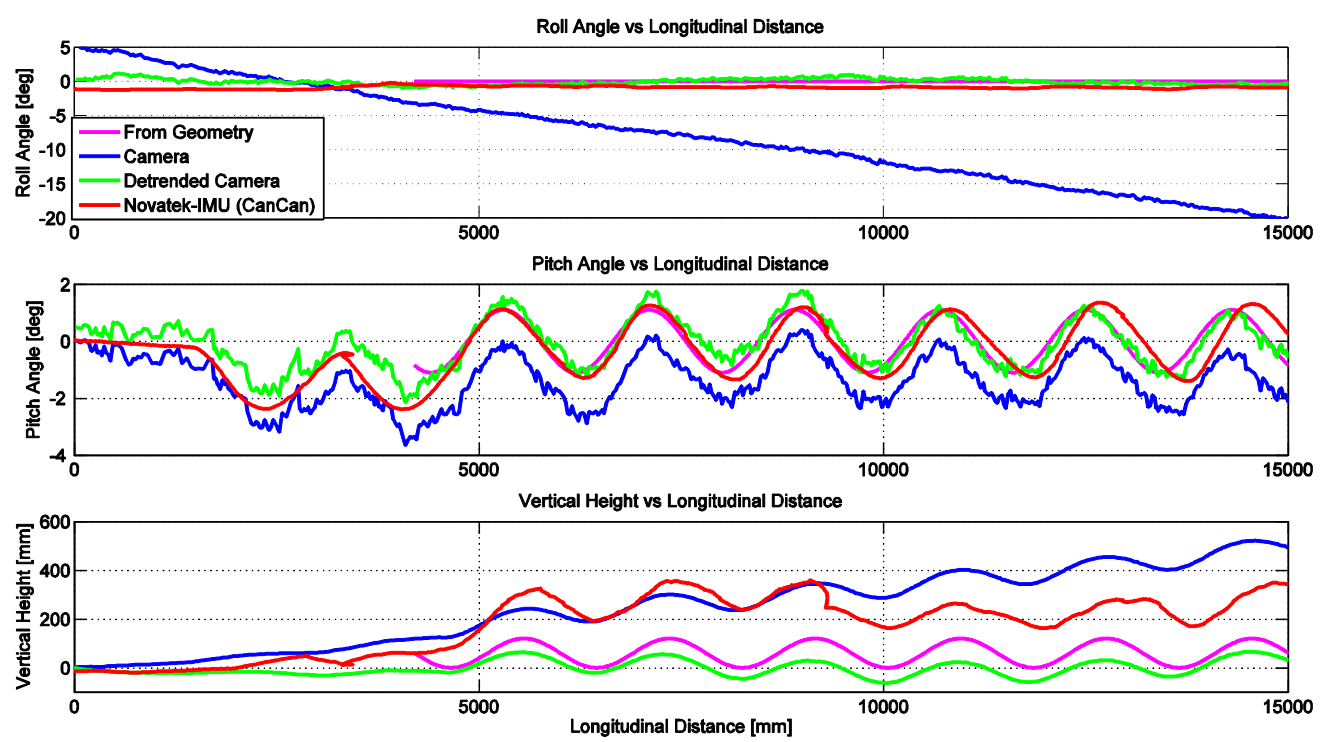

Figure 10 Measured roll, pitch and vertical height with pitch and vertical excitation

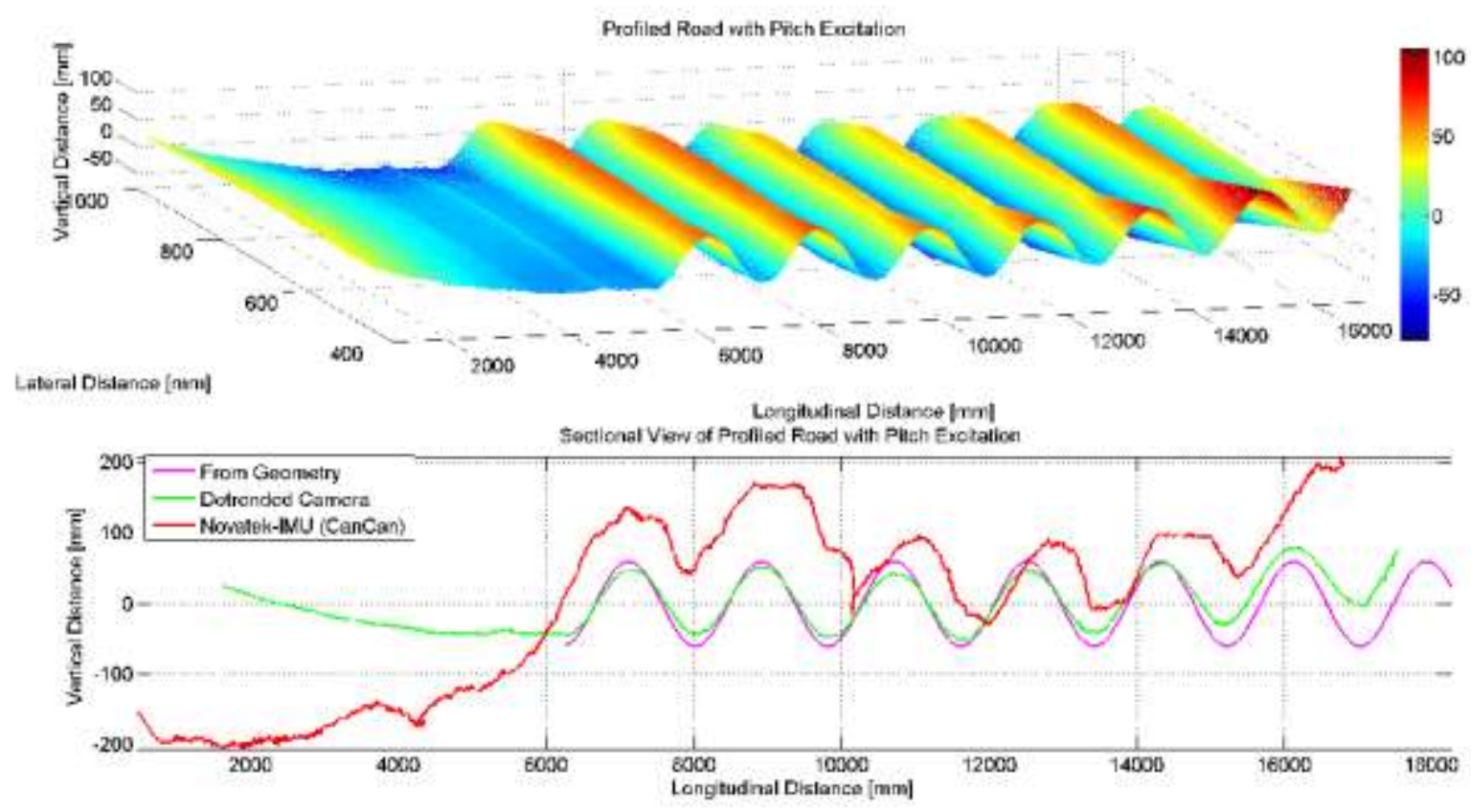

Figure 11 Measured 3D surface with sectional view of sinusoidal profile with pitch and vertical excitation 
Figure 11 shows the final profiled road. The 3D sinusoidal profile is shown in the first plot. The edges of the profile are affected by the extrapolation process and should therefore be disregarded. A sectional view of the profile from the camera based system is compared to the baseline profile and the CanCan machine in the bottom plot of Figure 11. This shows that the camera system provided better results than the CanCan system which was highly affected by the vertical height drift in the INS measurements. The amplitude obtained from the camera system is around $100 \mathrm{~mm}$ which is a $16 \%$ error on the $120 \mathrm{~mm}$ profile. However, it should be noted that this is still better than the INS system. The accuracy of the camera system could be improved by increasing the size of an individual surface from $300 \times 300 \mathrm{~mm}$ to a size which is comparable to the wavelength being measured. This however will result in a loss of measurement resolution if the camera resolution is not changed accordingly.

\subsubsection{Roll and Pitch Excitation}

In this test the road profiler underwent a roll and pitch excitation by having the left wheel follow a sinusoidal profile which is $180 \mathrm{deg}$ out of phase with the right wheels. This induces both a roll and pitch excitation at the same time while the camera system profiled a flat section of the track. Figure 12 shows the angles obtained from the camera as well as the INS. The angles from both systems follow the baseline profile well with the
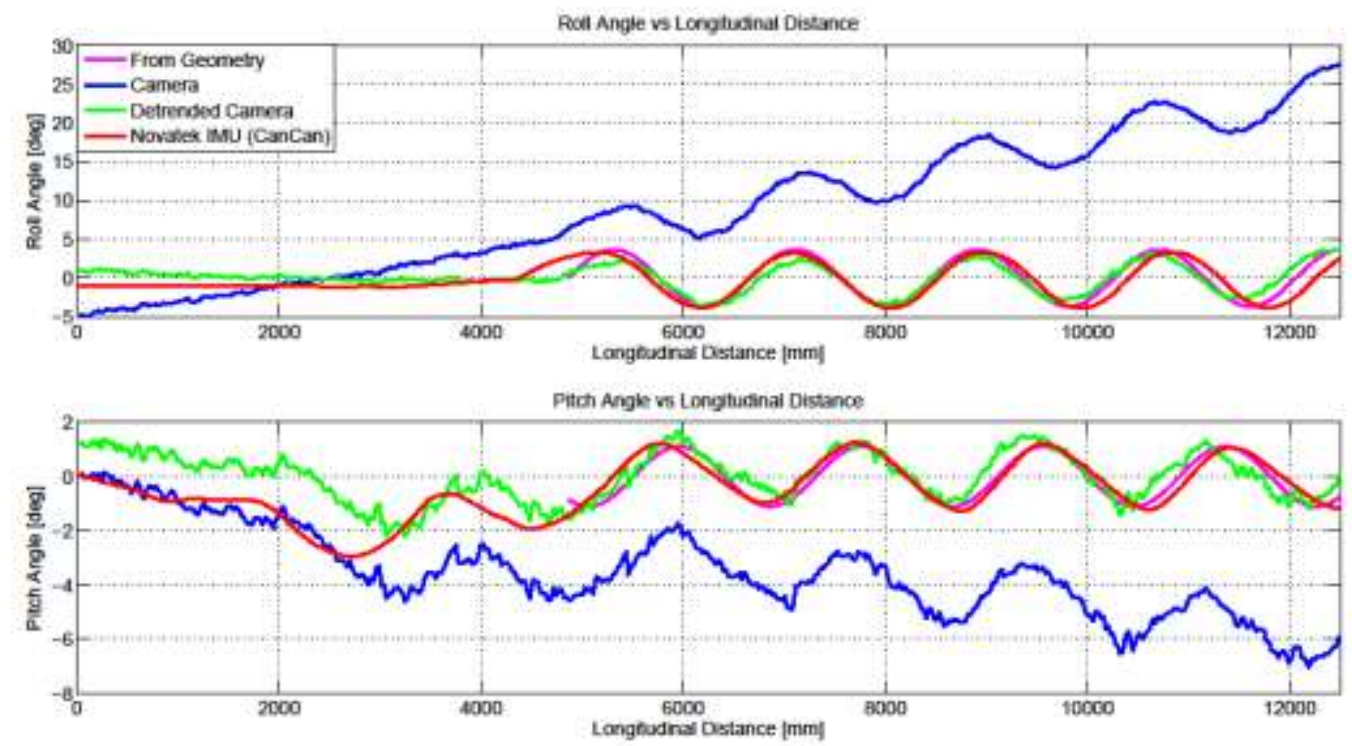

Figure 12 Measured roll and pitch angles with roll, pitch and vertical excitation

INS obtaining a slightly longer wavelength of the sinusoidal profile. The profiled road in Figure 13 shows that 
the profiled road remains flat, deviating a maximum of $20 \mathrm{~mm}$ from the ideally flat surface. The camera system therefore successfully removes the roll and pitch excitations.

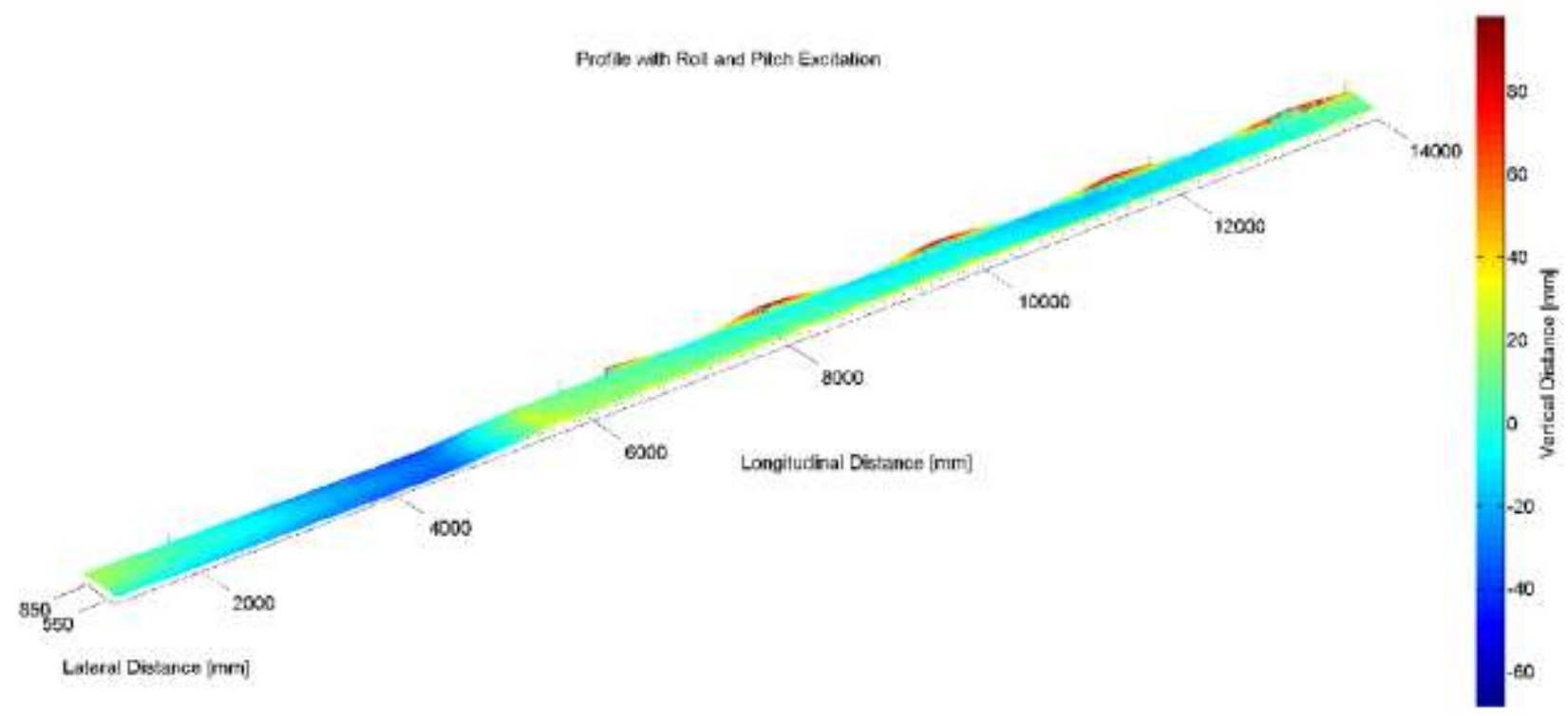

Figure 13 Measured flat surface with roll, pitch and vertical excitation
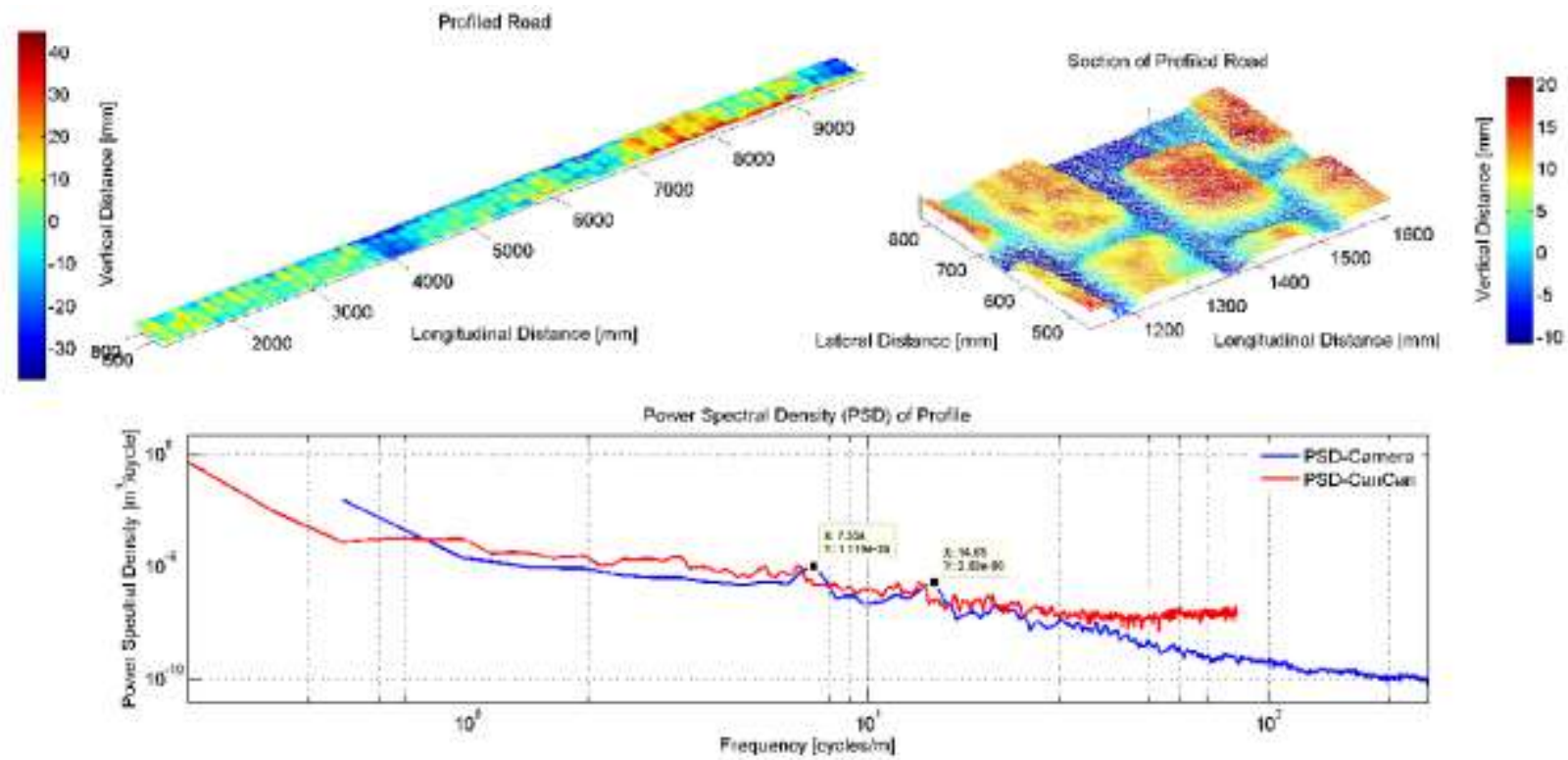

Figure 14 Measured 3D surface of Belgian paving. Zoomed in section of Belgian paving. Power spectral density of Belgian paving with proposed method and CanCan machine. 


\subsubsection{Belgian Paving}

A section of Belgian paving was profiled which contains much higher frequencies as compared to the previous terrains which were either flat or low frequency sinusoidal profiles. The profile obtained is shown in Figure 14. The figure shows a $10 \mathrm{~m}$ section of Belgian paving superimposed on a low frequency profile. A zoomed in section of the profile shows the resolution that can be obtained from the camera system, showing the bricks as well as spaces between the bricks very clearly. This method obtains better results than the CanCan machine which uses a road following wheel (stylus) with circular tip to measure the profile. The spatial resolution of the system tested can be easily improved by increasing the camera resolution without affecting the area measured. The diameter of the road following wheel effectively filters the profile depending on the radius of the stylus tip. A Power Spectral Density of the profile is given in the bottom plot. This plot shows that the camera method effectively captures up the two wavelengths at $7.32 \mathrm{cycles} / \mathrm{m}$ as well as $14.65 \mathrm{cycles} / \mathrm{m}$ corresponding to the average brick and spacing dimensions of $136 \mathrm{~mm}$ and $68 \mathrm{~mm}$. While the CanCan method does indicate that there is similar energies the peaks are much less pronounced and at lower spatial frequencies. The reason for the smaller peaks is partly due to the filtering effect but also due to the short section recorded at with a higher sampling spacing than the camera. The peaks are found at lower spatial frequencies since the stylus radius affects the measured profile at lower spatial frequencies indicating that the profiles are larger than actual. The study in [12] found that the system can successfully capture the spatial frequencies corresponding to the brick and spacing frequencies if the stylus radius is taken into consideration.

\subsubsection{Deformable Terrain}

In order to evaluate the potential of the proposed profiler to be used in terramechanics applications, a test was conducted over a deformable terrain. The terrain consisted of dry, course grained sand with no additional preparations conducted in order to allow the system to profile the terrain. A heap of the sand was deposited in front of one tyre of an off-road vehicle. The vehicle is equipped with off-road tyres which have $6 \mathrm{~mm}$ deep grooves. The road profiler (calibrated stereo rig) was mounted on the vehicle behind the right rear wheel (see Figure 15). A single tyre pass was performed while profiling the terrain.. 


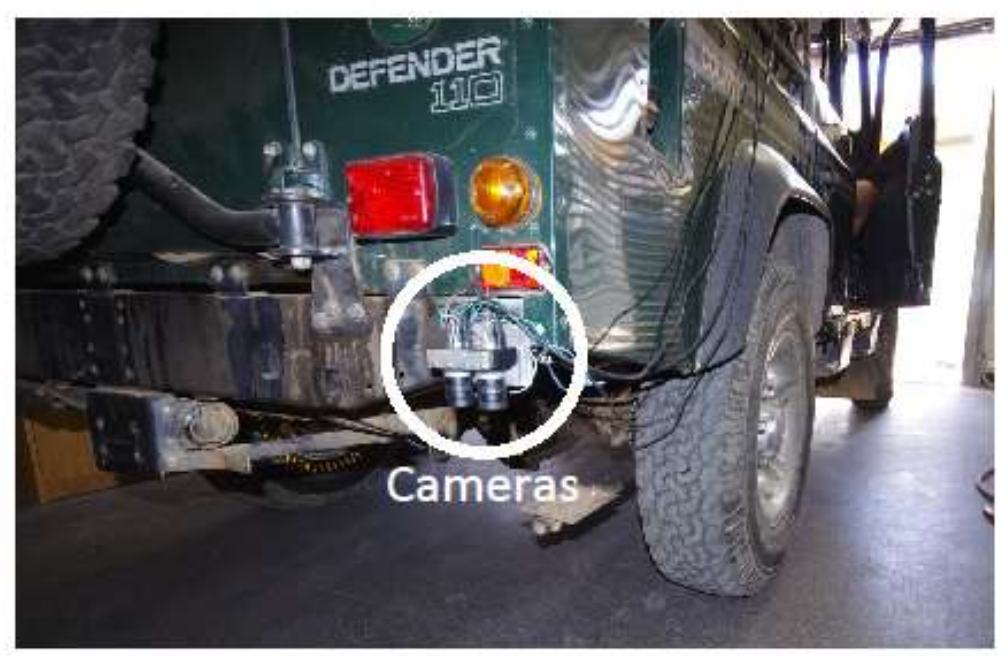

Fig 15 Deformable terrain test setup

The profiled terrain is shown in Figure 16. The left plot shows the full length of the profiled measured. The figure shows a small heap, superimposed on the heap are the tyre tracks. The measured profile is not perfectly flat but rather contains many low frequency oscillations on which the tread pattern are superimposed. In order to visualise the tyre tracks the lower frequency oscillations are removed by means of a fifth order polynomial detrending. The detrended profile is displayed in the middle plot in Figure 16. While the detrending removed most of the low frequency oscillations it was impossible to obtain to remove all without affecting the tread profile. Due to the small low frequency oscillations the colour In comparison a photo of the deformed soil is shown in the right most plot of Figure 16. The profiled terrain plot depicts the tyre tracks more clearly showing

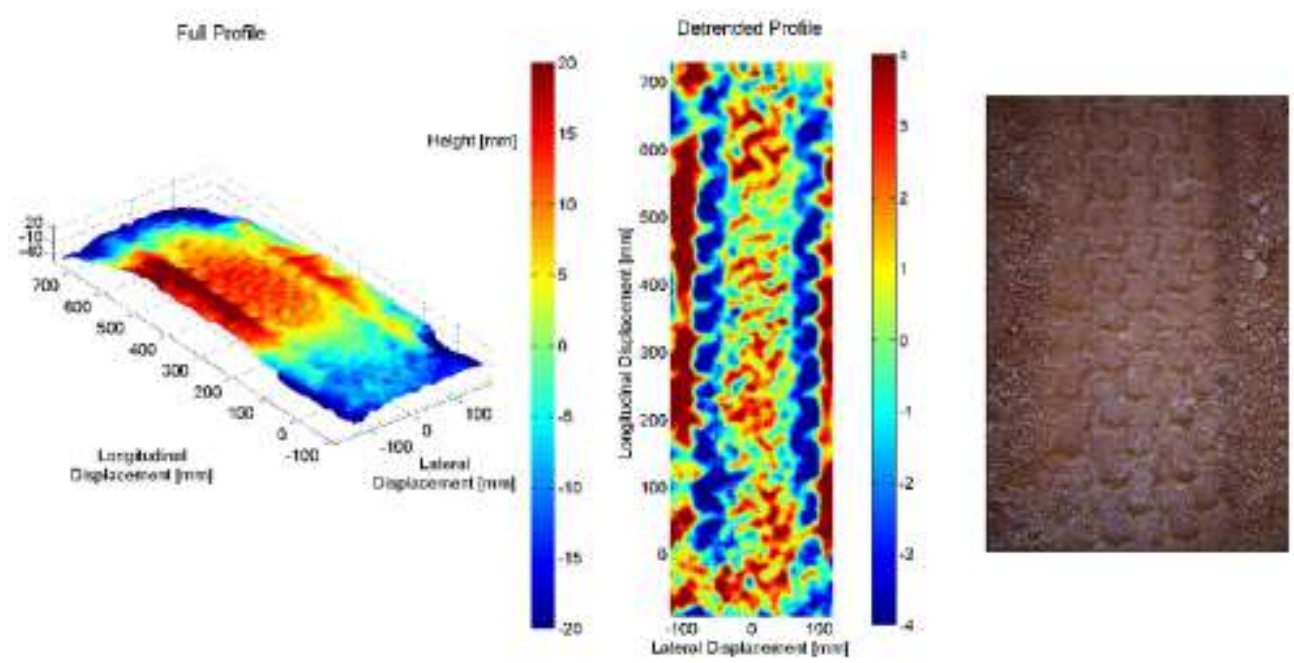

Fig 16 Measured deformable terrain profile 
the tread depth which corresponds to the $6 \mathrm{~mm}$ as measured on the tyre. The profiled terrain showing the tread also compares well to the photograph of the tread profile. The system thus successfully demonstrates the ability to measure a deformable terrain without any special treatment of the terrain.

\section{Future Work}

The developed system opens up possibilities in many areas of vehicle dynamics and terramechanics. The system provides an inexpensive technique to measure the profile of a terrain. As a result of the inexpensive nature and customisability of these sensors, multiple sensors can be added to vehicles. This will allow for example, in terramechanics not only the measurement of the rut depth before and after every wheel but also to obtain full 3D surface of the whole terrain before and after every wheel. This will allow for better development of off-road vehicle wheels with better traction and efficiency while providing a smaller impact on the environment.

The system also has the potential of being performed in real time by firstly utilising higher speed cameras to obtain frames at higher speed. The 3D depth and initial alignment phases can be performed in real time. Using Field Programmable Gate Array (FPGA) has yielded systems capable of performing the 3D depth mapping using more advanced algorithms, than presented here, at over 500 Frames Per Second (FPS) [34]. Thus, this system is capable of measuring rotational and translational rates at over 500 FPS. This can not only be used to measure terrain at higher vehicle speeds but also be used in other vehicle dynamics applications. The translational and rotational rates as well as positions and angles can be used to complement or provide a cheaper alternative to INS systems.

\section{Conclusion and Recommendations}

A road profiler system is which provides automatic continuous measurement of road profiles by making use of two cameras in a stereographic system is presented in this paper. The method of determining 3D profiles from a set of cameras is described. The camera motion is tracked using $2 \mathrm{D}$ image features along with their 3D coordinates yielding a fast camera pose technique. Once a certain distance of camera motion is reached a point cloud is captured which is aligned with the previous point cloud using 3D registration techniques. This yields 
multiple overlapping 3D point clouds. A novel interpolation strategy is presented which uses characteristics of the captured point clouds to increase the search region of an inverse distance to a power method. The presented method thus yields a system of which a regularised grid of the surface can be obtained at any resolution at a high computational efficiency.

The accuracy of the system is excellent, at the most basic setting, and can be improved by means of better cameras, lenses as well as changing the geometry of the cameras to each other as well as with respect to the road surface. It should be noted however that the computational efficiency decreases exponentially with an increase in camera resolution. The efficiency can however be improved with enchantments in the processing algorithms and implementation as well as by means of dedicated hardware. The measurements of the system, while containing drift, provides better accuracy and consistency than the GPS system employed by the INS system. While the GPS measurements can be improved by means of a RTK GPS, the overall positional accuracy is still questionable in providing millimetre accuracy required by road profilers. The camera system is shown to be able to remove all three translational and rotational disturbances from the system while allowing these disturbances to be measured as well.

However, since the registration method uses frames relative to one another to determine the relative motion it is impossible to obtain drift free estimates. While the drift can be reduced by proper calibration of the cameras a small amount of drift from noise and errors in calibration will lead to drift in the measurement over time. Possible solutions to this would be to use another set of cameras which always views fixed markers at some distance away. This would allow all frame motions to be corrected by means of motion determined from the same fixed points. The accuracy of this method is the largest concern as stereographic measurement accuracy deteriorates at greater distances. Another alternative which is frequently employed is to use an IMU/INS system to remove drift. Multiple algorithms can be employed to fuse the camera measurement with that of the IMU/INS [35], [36], [37]. These algorithms make use of Kalman filters to fuse the different INS and camera measurements together. While an IMU/INS is required to remove drift, the requirements of the system is much smaller than required for most road profilers. The system accuracy need not be very high, neither does it require a high sampling frequency. quent measurements, for example every 1 or $2 m$, would be enough to reduce the overall drift of the system. However if the system is equipped with GPS it would lend itself to the same problems that a conventional profiler has with dropped satellites, jumps in position etc. This may necessitate the development of another means of obtaining the position of the profiler from a fixed reference. The system also 
has the potential to vastly improve the field of terramechanics by providing an inexpensive sensor which can measure full 3D surfaces of the undeformed and deformed soil being worked by a vehicle. The system also has the potential to be implemented in real time to not only profile terrain at higher speeds but also to measure translation and rotational rates, positions and orientations.

\section{Notation}

\begin{tabular}{|c|c|}
\hline$d$ & Disparity \\
\hline$d(\overline{\boldsymbol{x}}, \boldsymbol{x})$ & Euclidean distance between points $\overline{\boldsymbol{x}}$ and $\boldsymbol{x}$ \\
\hline$I_{l}$ & Left image \\
\hline$I_{r}$ & Right image \\
\hline$I(x, y)$ & Image pixel at coordinates $\mathrm{x}, \mathrm{y}$ \\
\hline$P_{1}$ & Point set 1 \\
\hline$\overline{\boldsymbol{P}_{1}}$ & Mean of point set 1 \\
\hline$P_{1}^{i}$ & $\mathrm{i}^{\text {th }}$ point from point set 1 \\
\hline $\boldsymbol{R}$ & Rotation matrix \\
\hline$T_{I C P}$ & Transformation matrix from ICP algorithm \\
\hline$T_{\text {local }}$ & Relative transformation matrix between two clouds \\
\hline$T_{\text {total }}$ & Total transformation matrix relative to origin \\
\hline $\boldsymbol{t}$ & Translation vector \\
\hline $\boldsymbol{U}$ & Left SVD singular vector matrix \\
\hline $\boldsymbol{V}$ & Right SVD singular vector matrix \\
\hline$\Sigma$ & Singular values from SVD \\
\hline$w_{i}$ & Inverse to a power weighting value \\
\hline$x$ & Vertical image pixel coordinate \\
\hline$\overline{\boldsymbol{x}}$ & Point location in interpolated cloud \\
\hline$x$ & Point location in original cloud \\
\hline$x_{i, j}$ & Longitudinal position of point in point cloud matrix in row $i$ and column $j$ \\
\hline$x_{\text {step_ } i}$ & Longitudinal position increment per row increment ( $i$ direction) \\
\hline
\end{tabular}




\begin{tabular}{|c|l|}
\hline$x_{\text {step } \_j}$ & Longitudinal position increment per column increment $(j$ direction) \\
\hline$y$ & Horizontal image pixel coordinate \\
\hline$y_{i, j}$ & Lateral position of point in point cloud matrix in row $i$ and column $j$ \\
\hline$y_{\text {step } \_} i$ & Lateral position increment per row increment ( $i$ direction) \\
\hline$y_{\text {step } \_} j$ & Lateral position increment per column increment $(j$ direction $)$ \\
\hline$z_{i}$ & Vertical height of point $\boldsymbol{x}$ \\
\hline$z_{\text {interp }}$ & Interpolated vertical height \\
\hline
\end{tabular}

\section{ACKNOWLEDGEMENTS}

The financial assistance of the National Research Foundation (DAAD-NRF) towards this research is hereby acknowledged. Opinions expressed and conclusions arrived at, are those of the author and are not necessarily to be attributed to the DAAD-NRF.

\section{References}

[1] Andren P. Power spectral density approximations of longitudinal road profiles. international Journal of Vehicle Design, 2006;40, pp. 2-14.

[2] International Organization for Standardization ISO 8608: Mechanical Vibration - Road Surface Profiles Reporting of Measured Data, ISO 8608:1995(E).

[3] Shibly H, Iagnemma K, and Dubowsky S. An equivalent soil mechanics formulation for rigid wheels in deformable terrain, with application to planetary exploration rovers. Journal of Terramechanics 2005;42:1, pp. $1-13$

[4] Iagnemma K, Kang S, Brooks C, Dubowsky S. Multi-sensor terrain estimation for planetary rovers. In: Proceedings of the seventh international symposium on artificial intelligence. Robotics and Automation in Space, i-SAIRAS. 2003 
[5] Kurjenluoma J, Alakukkua L, Ahokas J. Rolling resistance and rut formation by implement tyres on tilled clay soil. Journal of Terramechanics 2009;46,:6, pp. 267-275

[6] Soane BD, Blackwell PS, Dickson JW, Painter DJ.. Compaction by agricultural vehicles: A review I. Soil and wheel characteristics Soil and Tillage Research 1980;1, pp. 207-237

[7] Soane BD, van Owerkerk C. Implications of soil compaction in crop production for the quality of the environment. Soil and Tillage Research. Soil Compaction and the Environment 1995;35:1-2, pp. 5-22.

[8] Anderson AB, Palazzo AJ, Ayers PD, Fehmi JS. Assessing the Impacts of Military Vehicular Traffic on Natural Areas. Journal of Terramechanics, 2550;42, pp.143-158.

[9] Spangler EB, Kelly WJ. General Motors Corporation. Research Laboratories. GMR road profilometer - a method for measuring road profile Warren, Mich. Research Laboratories, General Motors Corp., 1964.

[10] Kern JV, Ferris JB, Development of a 3-D Vehicle-Terrain Measurement System Part I: Equipment Setup. Proceedings of ISTVS: Innovations in Terrain and Vehicle Systems, ISTVS. 2007. Fairbanks, AK.

[11] Kertész I, Lovas T, Barsi A. Photogrammetric Pavement Detection System. International Society for Photgrammetry and Remote Sensing, Proceedings of the XXIst ISPRS Congress. 2008. Beijing.

[12] Becker CM, and Els PS. Profiling of rough terrain. Int. J. Vehicle Design,2014;64: 2/3/4, pp. $240-261$.

[13] Dhond U R, and Aggarwal J K.. A cost-benefit analysis of a third camera for stereo correspondence. International Journal of Computer Vision, 1991;6:1, pp.39-58.

[14] Zhang Z. . A exible new technique for camera calibration. Pattern Analysis and Machine Intelligence, IEEE Transactions on, 2000;22:11, pp. 1330- 1334.

[15] Konolige K. Small vision systems: hardware and implementation. Eighth International Symposium on Robotics Research, 1997, pp.111-116.

[16] Bradski G. The OpenCV Library. Dr. Dobb's Journal of Software Tools. 2000

[17] Xavier J. Sousa AMR, Morais JJL, Filipe VMJ, Vaz M. Measuring displacement fields by cross-correlation and a differential technique: experimental validation. Optical Engineering 2012;51:4

[18] Lowe D. Distinctive image features from scale-invariant keypoints. International Journal of Computer Vision, 2004;60:2, pp. 91-110 
[19] Bay H, Ess A, Tuytelaars T, van Gool L, Leuven BKU. Speeded-Up Robust Features (SURF). Computer Vision and Image Understanding 2008;110:3, pp. 346-359.

[20] Arun KS, Huang TS, Blostein SD. Least-Squares Fitting Of Two 3-D Point Sets IEEE Transactions On Pattern Analysis And Machine Intelligence. 1987;9:5

[21] Fischler MA, Bolles RC. Random sample consensus: A paradigm for model fitting with applications to image analysis and automated cartography. Communications of the ACM, 1981;24:6, pp. 381-395.

[22] Besl PJ, McKay HD.. A method for registration of 3-D shapes, Pattern Analysis and Machine Intelligence. IEEE Transactions on Pattern Analysis and Machine Intelligence. 1992;14:0.

[23] Rusinkiewicz S, Levoy M. Efficient variants of the ICP algorithm, 3-D Digital Imaging and Modeling, 2001

[24] OpenPCL [viewed 02/10/14 from http://www.pointclouds.org]

[25] Tombari F, Sailti S,, Di Stefano L. Performance Evaluation of 3D Keypoint Detectors International Journal of Computer Vision. 2013;102:1-3, pp. 198-220

[26] Zhong Y. Intrinsic shape signatures: a shape descriptor for 3D object recognition. In Proc. int. conf. on computer vision workshops. 2009, pp. 1-8

[27] Rusu RB, Blodow N, Marton Z, Soos A. Beetz M. Towards 3D Object Maps for Autonomous Household Robots. Proceedings of the 2007 IEEE/RSJ International Conference on Intelligent Robots and Systems. 2007. San Diego, CA, USA, Oct 29 - Nov 2,

[28] Wahl E, Hillenbrand U, Hirzinger G. Surflet-Pair-Relation Histograms: A Statistical 3D-Shape Representation for Rapid Classification. Proceedings of the Fourth International Conference on 3-D Digital Imaging and Modeling. 2003

[29] Rusu RB, Blodow N, Beetz, M. Fast Point Feature Histograms (FPFH) for 3D Registration. IEEE International Conference on Robotics and Automation. 2009. Kobe, Japan, May 12-17

[30] Detweiler ZR, Ferris JB. Interpolation Methods for High-Fidelity Three Dimensional Terrain Surfaces. Journal of Terramechanics, 2010;47:4, pp. 209-217.

[31] Smith H, Ferris JB. Calibration Surface Design and Validation for Terrain Measurement Systems. Journal of Testing and Evaluation (JTE). 2009;38:4

[32] Gerotek Test Facilities [viewed 08,09,14 from www.gerotek.co.za] 
[33] Novatel SPAN-CPT [viewed 08,09,14 from http://www.novatel.com/products/span-gnss-inertialsystems/span-combined-systems/span-cpt/\#overview]

[34] Georgoulas C, Andreadis I. A Real-Time Occlusion Aware Hardware Structure for Disparity Map

Computation. Image Analysis and Processing - ICIAP 2009 Lecture Notes in Computer Science. 2009;5716, pp. $721-730$

[35] Julier SJ, Uhlmann JK. Fusion of time delayed measurements with uncertain time delays. American Control Conference, 2005;6, pp. 4028 - 4033

[36] Zhang K, Rong XL, Zhu Y. Optimal Update With Out-of-Sequence Measurements. IEEE Transactions On Signal Processing. 2005;53:6, pp. $1992-2004$

[37] Li M, Mourikis AI. 3-D Motion Estimation and Online Temporal Calibration for Camera-IMU Systems. 2013 IEEE International Conference on Robotics and Automation (ICRA). 2013. Karlsruhe, Germany, May 610 